\title{
Making research evaluation more transparent: Aligning research philosophy, institutional values, and reporting
}

\author{
Michael R Dougherty \\ L. Robert Slevc \\ $\&$ \\ James A. Grand \\ University of Maryland
}

\begin{abstract}
There is a growing interest in changing the culture of psychology to improve the quality of our science. At the root of this interest is concern over the reproducibility of key findings. A variety of large-scale replication attempts have revealed that a number of previously published effects cannot be reproduced, while other analyses indicate that the published literature is rife with underpowered studies and publication bias. These revelations suggest that it is time to change how psychological science is carried out and increase transparency of reporting. We argue change will be slow until institutions adopt new procedures for evaluating scholarly activity. We consider three actions that individuals and departments can take to facilitate change throughout psychological science. These three actions are the development of individualized research philosophy statements, the creation of an annotated curriculum vitae to improve the transparency of scholarly reporting, and the use of a formal evaluative system that explicitly captures behaviors that support reproducibility. Our recommendations build on proposals for open science by enabling researchers to have a voice in articulating (and contextualizing) how they would like their work to be evaluated and by providing a mechanism for more detailed and transparent reporting of scholarly activities.
\end{abstract}

Keywords: Open Science, reproducibility, Research philosophy, Annotated curriculum vitae

Central to our motivation for writing this article is the current debate over reproducibility within psychological science and other fields, and related discussion regarding incentive structures for tenure and promotion. There is broad agreement that the incentive structure for tenure and promotion can reward research practices that incentivize novelty, expediency, and publication quantity and disincentivize practices that promote rigor, reproducibility, and scientific quality (e.g., Edwards \& Roy, 2017; Flier, 2017; Higginson \& Munafo, 2016; Nosek, Spies, \& Motyl, 2012; Smaldino \&
McElreath, 2016). This is not to say that rigor is not valued by researchers (Ebersole, Axt, \& Nosek, 2016), but rather that there is no widely-used reporting system that allows researchers to document their efforts at ensuring quality or facilitate evaluation of participation in robust scientific practices. To be sure, problems regarding reproducibility and incentives are multifaceted and cannot be solved with any singular reform. However, we believe there are many small "nudges" capable of better aligning the incentive and evaluation structures of our scientific 
community with the values and principles of robust science (e.g., Grand et al., 2018; Nosek et al., 2012). We further posit that changing culture and practices around these issues will involve empowering researchers to reflect upon and publicly express their views on how and why they conduct their research and then allowing them to report the steps they take to meet those expectations.

To this end, the goal of this article is to describe two interconnected practices that individuals and departments can take to encourage researchers to articulate their scientific values, incentivize good research practices, and empower researchers to change the way they do their work. These practices are (a) the creation of individualized research philosophy statements that reflect an individual's core research values and goals, and (b) the use of an annotated curriculum vitae (CV) for reporting research productivity and scholarship. We propose that giving faculty a voice in how their work should be evaluated and increasing transparency in the description of scholarship can encourage faculty to make choices in the conduct of their own research that will improve the quality of scientific output in a more bottom-up fashion. Further, we argue that the research philosophy statement and the annotated $\mathrm{CV}$ can facilitate hiring decisions by allowing departments to select applicants whose philosophy aligns with their institutional or departmental values; that they can improve equity and fairness in the evaluation of faculty for merit pay increases; allow tenure and promotion committees to better evaluate the scope of a candidate's contribution to science and reproducibility; and enable faculty to better reflect on one's own research progress in light of his or her philosophy.

\section{Understanding System Change: Culture, Context, and Criteria}

Research and theory in the psychological sciences has long acknowledged that the social systems in which persons and situations reside are capable of influencing our affect, behavior, and cognition (e.g., Lewin, 1947; Simon, 1956; Weick,
1976; 1995). An important theme emerging from this work is the recognition that facilitating effective change in organizational systems requires knowledge of its culture (i.e., broadly shared values, assumptions, and norms that define "good vs. bad," "acceptable vs. unacceptable," "desirable vs. undesirable," etc.), context (i.e., local practices, policies, reward systems, etc. that codify culture), and criteria (i.e., evaluative standards and related feedback systems reflecting how things are or should be done in ways consistent with the context; e.g., Cameron \& Quinn, 2011; Foster-Fishman, Nowell, \& Yang, 2007). In the context of nudging our scientific community toward more robust and reproducible scientific practices, lessons learned from organizational change scientists highlight the criticality of aligning distal cultural values around scientific quality and reproducibility with personal and proximal practices and procedures that support those norms.

Although the decentralized nature of most scientific disciplines and academia tend to render these concepts more diffuse and opaque, Figure 1 summarizes our conceptualization of these pillars in the context of research science: institutional values, researcher values, and transparent reporting of scholarship. Institutions such as one's department, university, and discipline play a significant role in conveying the values and expectations for faculty researchers (i.e., culture). However, these values and expectations cannot stand alone in guiding researcher behavior. Researchers need a way of expressing how their work aligns with institutional values (i.e., context) and a mechanism for reporting how their scholarship meets institutional expectations (i.e., criteria). Figure 1 also captures what we see as the relationships among these pillars. Without doubt, institutions and disciplines exert a strong top-down influence on the scientific values and practices which define its community; for example, universities set expectations for research productivity and enforce policies for how research productivity is reported and evaluated, while disciplines set scientific norms and standards. Though such institutions are slow to change and 
individual faculty (especially those pre-tenure) have few opportunities for exacting bottom-up change, feedback loops do exist from faculty and reporting mechanisms to these collective levels (the dotted lines).

To situate this framework within the conversation of robust scientific practices and promoting high quality science, consider the typical policies set by institutions for both research misconduct and appointment, tenure, and promotion. At the University of Maryland, the policy for research misconduct includes language emphasizing scientific integrity: "Integrity in research is the responsibility of the entire academic community. All members of the university community - students, staff, faculty and administrators - share responsibility for developing and maintaining standards to assure honesty, accuracy and objectivity in scientific and scholarly work and other creative activities and detection of abuse of these standards" (pg. 4, University of Maryland Policy and Procedures Concerning Research Misconduct, 2017). ${ }^{1}$ The policy defines misconduct as "fabrication, falsification, plagiarism, or any other practice that seriously deviates from practices commonly accepted in the discipline or in the academic and research communities..." (pg. 8) and questionable research practices as acts that "do not constitute misconduct or unacceptable research practices, but that require attention because they could erode confidence in the integrity of the research or creative activity" (pg. 8).

In terms of criteria for appointment, tenure, and promotion, the policy at the University of Maryland (University of Maryland Policy and Procedures on Appointment, Promotion and Tenure of Faculty, $2017)^{2}$ provides the following expectations for assistant, associate, and full professors (pp. 2-3):

https://president.umd.edu/administration/policies/sectioniii-academic-affairs/iii-110a

2

https://president.umd.edu/administration/policies/sectionii-faculty/ii-100a
Appointments at the Assistant Professor level. The appointee shall have qualities suggesting a high level of teaching ability in the relevant academic field, and shall provide evidence of potential for superior research, scholarship, or artistic creativity in the field. ...

Appointments at the Associate Professor level. In addition to having the qualifications of an Assistant Professor, the appointee shall have a high level of competence in teaching and advisement in the relevant academic field, shall have demonstrated significant research, scholarship, or artistic creativity in the field and shall have shown promise of continued productivity ...

Appointment at the Full Professor level. In addition to having the qualifications of an Associate Professor, the appointee shall have established a national and, where appropriate, international reputation for outstanding research, scholarship or artistic creativity, and a distinguished record of teaching. ... 


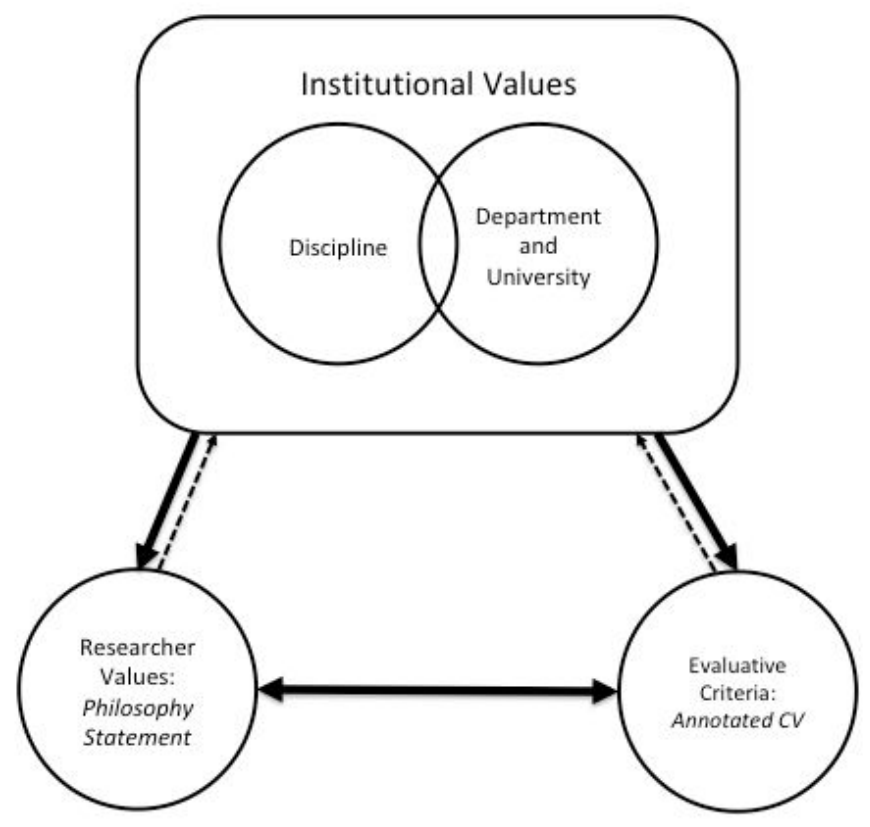

Figure 1. Schematic of the three pillars of the conduct of science.

We suspect that these promotion, tenure and misconduct policies (or their equivalent) are similar to how many colleges or universities tend to express such values ${ }^{3}$. Both policies offer few specifics regarding what constitutes 'superior', 'significant', or 'outstanding' research (in the case of promotion and tenure) or what constitutes questionable and unacceptable practices (in the case of research misconduct). Nevertheless, they do broadly characterize the normative standards/statements that reflect key institutional values (integrity, superior research, productivity, etc.).

In many cases, the onus is placed on individual departments to provide more detailed definitions of quality. Unfortunately, many departmental criteria for tenure and promotion also lack specificity. For

\footnotetext{
${ }^{3}$ It bears mentioning that different universities or colleges may hold different values, depending on the specific mission of the university or college.
}

example, promotion and tenure standards for Associate professors in our department call for "evidence of accomplishment" in publishing in prestigious journals; national recognition in an area of research; and peer recognition as indexed by citation counts and impact factors (amongst other descriptors). However, we note that even at this more constrained level, there is little specific guidance on how to define scientific quality. The few criteria often invoked as indices of such quality--citation and impact factors--are imperfect at best (for discussion see, e.g., Arruda et al., 2016; Hicks, Wouters, Waltman, Rijcke, \& Rafols, 2015; Verma, 2015; Declaration on Research Assessment, http://www.ascb.org/dora/). Further, what constitutes a questionable research practice is of current debate, and there are no agreed upon rules for differentiating questionable practices from those that are accepted, but discouraged. For example, 
some practices, such as collecting more data after failing to obtain a significant result or selectively excluding (or including) extreme data points are sometimes seen as defensible and are commonly used (Agnoli et al., 2017; John, Loewenstein, \& Prelec, 2012; Yu, Sprenger, Thomas, \& Dougherty, 2014).

Given that these gray areas can be difficult to define collectively, we believe one viable solution is to allow faculty the opportunity to express their own scientific values, contextualize their individual approach to science, and report more details about individual pieces of scholarship than are common in current practices. We believe a first step toward achieving this goal is for researchers to reflect upon and explicitly describe the context in which their research is conducted and how/why this reflects desirable cultural/institutional values. Though there have been recent high-profile cases of research misconduct within the psychological and brain sciences (e.g., Diederik Stapel), we believe that most scientists embrace or want to embrace practices that will ultimately enhance the reproducibility of their science. However, we also believe many researchers feel that (a) they have no mechanism for expressing their core scientific values and advocating for how those values guide their research practices, and (b) institutional incentives and criteria for tenure and promotion serve as barricades as opposed to buoys to instituting good practices. We believe that in enacting change to incentivize better research practices, researchers should be given the opportunity to more completely communicate their specific research values and goals.

A second critical piece for achieving this goal involves improving and encouraging transparency of academic reporting. Nosek et al. (2015) developed the Transparency and Openness Promotion guidelines as a way of changing journals' publication policies to incentivize greater use of open-science practices; however the practices encouraged by the Transparency and Openness Promotion guidelines are not linked explicitly to faculty reporting mechanisms. For instance, at research universities, dossiers submitted for promotion and tenure review typically include information on one's program of research (the CV, sample publications, the research statement), and detailed information on one's teaching philosophy and effectiveness. Though dossiers are intended to enable evaluators to assess the quality and extent of one's research productivity, they ironically lack information pertinent to assessing individual pieces of scholarship (aside from a handful of sample articles). Evaluators are typically forced to over-rely on imperfect metrics such as number of publications, journal impact factors, reputation of publication outlets, and citation rates. These indices do not adequately capture nuances in one's research efforts and impact and can conceivably even propagate subtle and difficult to detect biases. For example, implicit racial or gender biases can adversely affect citation rates (Chakraverry, Kuo, Grubbs, \& McIlWain, 2018; Knobloch-Westerwick, Glynn, \& Huge, 2013; Maliniak, Powers, \& Walter, 2013). In an analysis of gender bias, Maliniak et al. (2013) found that female authored papers were cited significantly less often than male-authored papers, even after controlling for a variety of potential confounds. Data also indicate that women are less likely to engage in self-citation, which itself actually affects the rate of future non-self citations (Fowler \& Aksnes, 2007). Thus, not only are men more likely to cite their own work, but the self-citations can actually exacerbate the gender-bias. On the flip side, there is evidence that simply being a graduate from "Dr. Big Shot's" lab (Ball, 2011) and posting preprints (Schwarz \& Kennicutt, 2004) can increase citation rates. Journal impact factors and h-indices, which rely on citation counts, have also been criticized for the potential for being gamed (e.g., see Caon, 2017; Edwards \& Roy, 2017). For instance, the impact factor can be artificially inflated by editorial practices that encourage authors to cite other articles from the journal to which they are submitting. Focusing on number of publications may also be problematic because it can promote the use of underpowered studies (Higginson \& Munafo, 2016) and 
"propagate poor research methods and abuse of statistical methods" (pg. 2, Smaldino \& McElreath, 2016; see also Edwards \& Roy, 2017). Although the merits and problems with these indices are complex (for discussion see, e.g., the recent special sections on scholarly merit in psychological science from volumes 11 and 12 of this journal), all of these examples highlight that metrics based on citation rates and publication numbers are fallible at best (and misleading at worst) indices of scientific quality and impact. Citation rates, h-indices, and journal impact factors may well serve as a proxy for readership, however we argue that they are not good proxies for research quality.

In what follows, we describe how we believe these currently missing pieces in the academic system could be addressed through the adoption of research philosophy statements and annotated CVs in academic departments. We discuss the relation of these two elements to departmental review criteria and the role of reproducibility in both the development of research philosophy statements and annotated CVs. We additionally include suggestions for developing a research philosophy statement and choosing dimensions for an annotated CV (examples of each are provided online, https://osf.io/gp5qt/).

\section{Research Philosophy Statement}

It is common for newly minted $\mathrm{PhD}$ students as well as faculty to create Statements of Teaching Interests and Research Interests. These statements are intended to articulate in a concise manner one's general approach to teaching and research. One major difference between these two documents, however, lies in their philosophical orientations. Teaching statements are frequently organized around one's teaching philosophy. The teaching philosophy is a statement about what one values in teaching along with his or her pedagogy, which reflects and guides the actions one takes in the classroom. In contrast, the construction of most research statements are, in our experience, much less focused on values and philosophy and more focused on describing one's past research, ongoing projects, and future plans. However, we believe that research statements can and should also be based on a set of core values in a manner similar to how one creates a teaching philosophy. In the same way that articulating one's teaching philosophy is likely to motivate proactive changes to align behaviors with values, we argue that a well articulated research philosophy can nudge one to take concrete steps toward ethical and reproducible research, help communicate how one wishes his or her work to be evaluated, improve training of future scientists, and enable one to reflect on (and easily communicate) his or her unique contribution to the department, university, and discipline.

The first step to developing a research philosophy is to identify which specific categories to emphasize when describing one's approach to conducting and participating in scientific research. There are a variety of categories that could be included, but the key is to document information that reflects the ways in which one conducts and contributes to science through his or her research. Box 1 provides examples of five broad categories and related points that could be included in a research philosophy, including type of scholarship, data sources, knowledge dissemination goals, perspectives on reproducibility and open science, and authorship philosophy. Additionally, these categories help further emphasize the many different ways in which research can be conducted. For example, some researchers value tackling broad conceptual problems, whereas others may prefer to address specific empirical questions. Some researchers prefer research that has immediate translational value, whereas others focus on basic laboratory findings that advance foundational knowledge. Finally, some researchers may let their research be driven more by the tools they have at their disposal and address a wide range of questions afforded by those tools, whereas others may be

\footnotetext{
${ }^{4}$ Although our focus is on the role of a research philosophies within academia, we believe that writing and maintaining a research philosophy can be useful in any type of research-oriented career.
} 
more motivated by theoretical questions and use tools as a way to address those questions.

Although researchers likely contribute in many ways using a variety of approaches, there are also likely stable individual differences. Importantly, differences across faculty in their approach to science can lead to very different looking CVs, even if the body of work itself is essentially identical. For example, some faculty may expect to be included as authors on publications based on student dissertations and theses, whereas others may view dissertations and theses as providing an opportunity for students to publish independently. Some faculty participate in large collaborative networks and gain authorship on many papers for which their individual contributions may be important, but more diffuse; others may choose to work with only a few collaborators but play a larger role in each publication. Some labs may institute policies that require internal replications and open science practices, whereas other labs may not follow such practices. All of these choices involve trade offs -trade-offs that are reflective of one's general approach to science. Diversity in both approach and practice can and should be valued to promote robust science (Grand et al., 2018), but we lack methods for explicating and making the value of these differences understood by evaluators and members of our scientific community. We believe that allowing researchers to succinctly summarize their approach to conducting science provides a critical and needed outlet for researchers to provide information on how their research is conducted rather than only what is produced.

Of final note, it is important to not only describe what one's core values are in the research philosophy, but also why those are important for one's work. Recall that a primary purpose of the research philosophy statement is to provide context to one's scientific work in a manner that links practices to institutional values. Reflecting on and providing information about both how and why an individual conducts research in a particular manner serves two goals. First, it provides the researcher an opportunity to articulate a "vision" for their research process (i.e., how one carries out scientific research rather than the outcomes of that research per se) and identify the strategies they use (or strive to use) to achieve that vision. Notably, this is consistent with best practices in performance management systems promoted in the organizational sciences which emphasize the importance of employee voice, commitment, and engagement in establishing collectively agreed upon performance criteria (Aguinis, 2013; Aguinis, Joo, \& Gottfredson, 2011; Den Hartog, Boselie, \& Paauwe, 2004). Second, it provides institutional evaluators and members of the scientific community critical information about the ways in which researchers carry out research activities that is not -and cannot be -- adequately reflected by current metrics of research productivity. This point implicitly recognizes the critical difference between evaluating scientific quality in terms of performance-as-results (i.e., number of publications, impact factor of publications) versus performance-as-behaviors (i.e., what researchers do to generate research outputs; e.g., Beck, Beatty, \& Sackett, 2014; Campbell, 1990; Viswesvaran \& Ones, 2000). Changing researcher behavior will only happen if behavior is what is valued. Continuing to emphasize what we do/produce while wishing to change how we do/produce is nothing more than the folly of rewarding A while hoping for B (Kerr, 1975). 


\section{Box 1. Sample categories and examples of those categories to consider including in a research philosophy.}

What type of scholarship do you focus on?

- To what extent does your work focus on major theory development, paradigmatic empirical work, and/or methodological innovation?

- Does your work involve computational or quantitative modeling? If so, why?

- To what extent do you aim to contribute to basic science, policy, and/or practice?

- To what extent do you prefer to synthesize and comment on existing research versus generate new data?

What characterizes your data sources?

- To what extent does your work contain original data, re-analyzed previously published data, or use archival data sources?

- What type of data do you work with (neuroscience, longitudinal, laboratory, clinical, internet)?

- What populations does your work focus on (children, clinical, aging) and why?

What are your publication and knowledge dissemination goals?

- To what extent do you prefer to publish more concise papers with a smaller number of experiments or more comprehensive work that involves multiple experiments?

- $\quad$ Do you prefer to publish theory papers separate from empirical pieces or together?

- When working with large multivariate datasets, do you prefer to publish multiple papers based on different aspects of the data or do you publish comprehensive papers that report on all aspects of the data?

- Do you view non-standard disseminations such as blog posts and press releases as part of your job?

What do you do to ensure reproducibility of your work?

- $\quad$ To what extent do you make data and methods easily accessible to others?

- What steps do you take to ensure statistical analyses are robust and verifiable?

- What is your perspective on replication and pre-registration?

- How do you view exploratory analyses?

What is your philosophy regarding authorship?

- How do you decide who deserves authorship and on author ordering?

- Do you maintain large networks of collaborators with whom you tend to publish, or do you tend to work with just a few individuals?

- What is your preferred authorship role in student projects, including theses and dissertations?

In sum, articulating a clear research philosophy allows researchers to state their values and research goals as well as indicate how their research activities align with the values of a department, university, and discipline. It also better enables researchers to reflect upon what they do well, and identify specific elements of their research approach that may need greater attention. A research philosophy that addresses some or all of the above categories also increases the transparency of one's research goals and enables evaluators to better contextualize the scope of one's scholarly activity. This latter point implies a need to link the research philosophy to appropriate indicators in a transparent 
way so that individuals (and evaluators) can evaluate his or her progress in light of the stated philosophy and goals. Below, we illustrate how this can be done through the use of an annotated CV.

\section{Annotated Curriculum Vitae}

Though the research philosophy may be useful by itself, it is also important to consider methods for evaluating how well one's research output conforms to the values stated in the philosophy. That is, we believe there needs to be a parallel mechanism for "measuring" or documenting how well one achieves the values espoused in a research philosophy. We suggest that the $\mathrm{CV}$ is one appropriate and underutilized place for this. The CV serves as a summary document of one's job experience and includes items such as education, awards, grant activity, service activities, and evidence of scholarship. Each of these items can be, and sometimes are, annotated. For example, the entry for education may include recognition for honors, the researcher's specialty area, and the title of one's thesis or dissertation. Grants often include information regarding the funding agency, priority score, funding amounts, title, and a brief description. Surprisingly, however, researchers rarely annotate individual pieces of scholarship, even though doing so could be enormously helpful for articulating the scope and unique contribution of their published work. We propose that annotating individual publications can serve the broader goal of connecting one's scholarship to both his or her research philosophy and to institutional or departmental values.
Evaluations of faculty typically do (and should!) consider more than research contributions, but research productivity measured in terms of number of publications is clearly a major factor for research-oriented universities. The standard $\mathrm{CV}$ in most dossiers includes a list of published papers, usually broken up into peer reviewed research articles and non-peer reviewed papers. Papers are typically listed on the $\mathrm{CV}$ in citation format, with little to no information pertaining to the type of paper, the scope of the research/scholarly activity, or effort undertaken for ensuring reproducibility. We propose a simple modification to this format to explicate the unique contribution of each paper along a set of agreed upon dimensions. We suggest that the number of dimensions remain relatively small so as to keep the process from becoming overly burdensome. While there are many relevant dimensions that a department may wish to capture on an annotated $\mathrm{CV}$, we recommend the seven dimensions listed in Box 2 as starting points. Of course, exactly what should be included on an annotated CV should be decided upon by individual departments/institutions and should reflect that institution's specific values. For reference, for the annotated format in Box 2, we found that it takes no more than 1-2 minutes per article to document these details, and note that some of these proposed dimensions are already material required by some journals. The annotated $\mathrm{CV}$ could also easily incorporate "badges" earned for open-science practices (cf. Kidwell, et al. 2016).

\section{Box 2. Example details for annotation of individual research papers.}

Article type: review/theoretical/empirical/quantitative/commentary

Data: Original/previously published/archival/NA

Number of experiments and sample size of each: $\operatorname{Exp} 1(\mathrm{~N}), \operatorname{Exp} 2(\mathrm{~N})$

Data type: behavioral/neuro/longitudinal/internet/laboratory/clinical/simulation/unique sample characteristics

Reproducibility: no comment/includes replication/open data/open method/conducted robustness or sensitivity analysis/cross validation \{provide open science link if possible\}

Authorship Role: conceptualized problem/wrote original draft/edited draft/collected data/analyzed results/supervised student thesis or dissertation/scope of collaboration 
Contribution: what is the unique contribution of the article to science?; what features of the article are you most proud of?; how does this relate to the research philosophy?; were there any unique challenges involved in this work? Were novel methods created?

See https://osf.io/gp5qt/ for a more in depth justification for each of these criteria.

The benefit of the annotated $\mathrm{CV}$ is that it highlights the types of trade-offs in research and publication that are routinely made and which presumably are guided by one's research philosophy/values. For instance, an annotated CV that indicates the number of experiments in a paper and the number of subjects per experiment enables evaluators to better take into consideration the scope of one's work, and make better comparisons to disciplinary norms. In some contexts, five single-experiment papers might be functionally equivalent to one or two multi-experiment papers in terms of the scope of the work; in other contexts a single-experiment paper may be equivalent to a multi-experiment paper (e.g., longitudinal data, client/case study work). The annotated $\mathrm{CV}$ also enables researchers to better document how their research output fits within their own research philosophy as well as their contributions to science and/or outreach. That is, it allows researchers to provide more complete criteria for evaluating and understanding the nature of their work. Authors who value fewer more inclusive pieces of work over multiple more concise contributions will not feel disincentivized to pursue this approach if they know that the effort will be recognized. Those who make an explicit effort to ensure reproducibility will not feel penalized for the extra effort required for using open science practices, conducting replications, gathering larger samples, or conducting sensitivity or robustness analyses, and may even feel incentivized if those efforts are made transparent to evaluators. Moreover, once these efforts are documented in the annotated $\mathrm{CV}$, the tradeoffs inherent in ensuring reproducibility can be explicitly modeled by evaluators by using compensatory decision algorithms (see the "Implementation" section below for a specific suggestion). In short, the annotated $\mathrm{CV}$ serves a critical role by closing the feedback loop between departmental/institutional and researcher values. It not only enables one to capture what research is being produced, but to again showcase how and why that work aligns with and contributes to the goals and values of one's scientific community.

Note that the article details in Box 2 do not focus solely on reproducibility, nor are all aspects of reproducibility easily captured by these dimensions. Even the reporting of sample size can be difficult to interpret without knowledge of the experimental design. However, the details add information over current practice that should allow evaluators to better understand the type of product, scope of work, and overall contribution. It also provides faculty with a way of documenting extra steps that they might have undertaken but that are not evident in the final products, such as an unpublished replication, additional analyses that were conducted on the data to verify the results, cross-validation, or quantitative modeling efforts. Recognizing that there are many ways to do good science and improving transparency of reporting should empower faculty to take steps to improve their own science.

Although not all of these details focus on reproducibility, the annotated CV certainly does allow researchers to specify what they do to enhance the reproducibility of their work. Goodman et al. (2016) identify four categories of reproducibility: methods reproducibility, reproducibility of results (i.e., replication with corroborating data), robustness and generalization, and inferential reproducibility. Activities that support these four types of reproducibility are all important for the conduct of science and should be documented as part of one's scholarly activity. 
Blanket statements that one values reproducibility or badges denoting the use of open science approaches or pre-registration do not capture the variety of ways in which one may enhance the reproducibility of their own work. We believe that the annotated $\mathrm{CV}$ allows one to document these efforts in a more complete way and for individual pieces of scholarship. Adopting a broad definition of reproducibility and encouraging people to document those efforts is important for shifting both the incentive structure and our scientific culture toward one that ascribes greater value to scientific transparency and robust science.

\section{Suggestions for Implementing the Philosophy and CV Changes in Your Department.}

An important question in considering our proposal concerns the issue of how and when the research philosophy and annotated CV can be used. Although the Philosophy and $\mathrm{CV}$ are complementary documents, we believe that they should be used in different ways. We propose that the Research Philosophy statement be used in major milestones such as appointment, pre-tenure review (at the University of Maryland this occurs once before tenure review in year 3), tenure/promotion reviews, and post-tenure review. In contrast, we suggest using the annotated $\mathrm{CV}$ as part of the annual review process as well as including it as part of the review process at major milestones. Including the annotated $\mathrm{CV}$ in annual reviews incentivizes behaviors that have been explicitly endorsed by the faculty, as suggested below, while also increasing the transparency by which faculty are evaluated. Pairing the annotated CV with the research philosophy at major milestones provides faculty reviewers with a richer set of information on which to evaluate faculty. This can be particularly important for early career (pre-tenure) reviews where corrective feedback can help individuals bring their body of work more in line with departmental expectations.

Box 3 summarizes our recommendations for constructing research philosophies and annotated $\mathrm{CV}$. We believe a critical key to success for this process will be to ensure that individual departments solicit input from their faculty to inform the creation of department-specific values and annotation formats. Additionally, and perhaps more importantly, than this is developing a process by which information recorded on an annotated CV--and documents a researcher's efforts to achieve these criteria--feeds into the review process. Although there may be many ways to incorporate this data into evaluations, the process ought to be transparent and reproducible. One approach that satisfies both of these criteria is Multi-Attribute Utility analysis, which is a formal decision analytic tool. The Multi-Attribute Utility analysis method involves identifying relevant evaluative dimensions, assigning importance weights to those dimensions, rating individuals on each dimension independently, and deriving a weighted aggregate score for each evaluatee. There are several benefits of the Multi-Attribute Utility analysis. First, it requires specification of the criteria on which faculty are evaluated. By definition, this necessarily increases the transparency of the evaluative process - faculty know precisely what they are being judged on and the relative importance of each of the criteria. Second, criteria are evaluated quantitatively and combined using a linear model to form an overall evaluation. This promotes a holistic evaluation process that is verifiable and reproducible from evaluation ratings. Third, because Multi-Attribute Utility analysis uses a linear model to combine ratings across evaluative criteria, it explicitly models the types of trade-offs researchers are often forced to make.

This later feature is particularly important for addressing one potential fear that faculty may have regarding the inclusion of open-science practices and efforts that support reproducibility on annual reviews. We suspect that some faculty may perceive that they will be unfairly treated or penalized for not partaking in these activities. However, faculty can only be penalized if open science practices are treated in a non-compensatory way. The use of a compensatory decision algorithm such as Multi-Attribute Utility analysis averts this criticism 
by explicitly modeling tradeoffs. So long as researchers offset their lack of effort in engaging in open science practices with increased effort on other metrics of productivity, then there is no inherent penalty for not engaging in open science practices; by the same token, there is no inherent penalty for engaging in open science practices. Multi-Attribute Utility analysis explicitly recognizes the trade-off between number of publications and the time/effort needed for increased transparency (open science practices) and efforts that enhance reproducibility. So long as the Multi-Attribute Utility analysis includes multiple indicators of productivity and reproducibility, faculty should have no reason to worry about being unfairly penalized for not engaging in open science practices. However, we also believe that explicitly modeling the trade-off between reproducibility and quantity of publication can nudge researchers away from focusing exclusively on maximizing the number of research publications. Allowing for a trade off between activities that we assume promote better quality research (i.e., reproducibility efforts) and quantity of research output should in principle encourage individuals to optimize their true scientific productivity while reducing incentives for bad actors who exploit a system that overemphasizes research quantity. ${ }^{5}$ Despite these points, we believe that implementation of this type (or any type) of evaluative system should not be

\footnotetext{
${ }^{5}$ Edwards and Roy (2017) suggest an inverted-U shaped function relating true scientific productivity to trade-offs in quality and quantity of publications, where "true scientific productivity" is defined as the accumulation of true scientific facts. Over emphasis of quantity is assumed to lead to an increase in erroneous (non-factual) results, even without assuming questionable research practices (and hence a decreasing rate of true science productivity), whereas an overemphasis on quality necessarily hinders rate that true scientific facts are identified and entered into the published record.
}

taken as a license to target individuals who choose to conduct their research differently.

A sample Multi-Attribute Utility analysis sheet is provided on the accompanying Open Science Framework page (https://osf.io/gp5qt/). This Multi-Attribute Utility analysis covers three broad categories of evaluation (research, teaching, and service). A dimension such as Transparency and Reproducibility Efforts is informed by the use of open science practices and efforts to engage in other activities that might enhance the reproducibility of particular findings (e.g., such as conducting replication studies, robustness/sensitivity analysis, cross-validation, etc). In contrast, a dimension such as Quality and Scope of Publications might be informed by details such as number of experiments and sample size in individual papers. Factors in this category should be evaluated relative to disciplinary norms and the methodological practices employed. For example, the purpose of including sample size in our analysis is to explicitly incentivize faculty to conduct higher-powered studies rather than disincentivize research examining special populations (e.g., unique clinical settings) or restrictive levels of analysis (e.g., organizations, cultures, etc.).

A critical question is how to set the weights used in the Multi-Attribute Utility analysis. In keeping with our proposal that faculty have input into how their work is evaluated, we propose soliciting weights from individual faculty and aggregating across faculty using the arithmetic mean. This process achieves two related goals. First, it ensures that individual faculty have a say in how their work is evaluated as a factor of $1 / \mathrm{N}$. Second, it enables individual faculty to observe what their colleagues view as important. This latter point is important because it enables individual researchers to make informed decisions about how to conduct their own research in light of how that research is being evaluated. Sharing assigned weights across faculty (perhaps de-identified) can both increase transparency of the evaluation process and serve as a feedback loop for faculty. Moreover, it allows faculty to better evaluate how their own 
research approach fits with departmental values and norms.

As departments begin developing plans to adopt an annotated $\mathrm{CV}$, research philosophy, and changes to their evaluation processes, it may be worth considering a phased implementation. One easy place to start is making the annotated CV optional in annual reviews and progressively move toward the goal making them a requirement. Similarly, input can be solicited for the development of the evaluation framework (i.e., dimensions \& weights for a Multi-Attribute Utility analysis) that can be initially used only for self-evaluation, and then later phased into department-level evaluations. We would also urge proponents of our proposals to recognize that the adoption of open science practices and the implementation of practices that enhance reproducibility should (in our view) neither be seen as a mandate nor be expected to occur instantaneously. It will take time for faculty to change their research pipeline and laboratory practices. The key point is that so long as departments and universities change their incentive structures, faculty will eventually bring their practices in line with the new norms. The status quo of talking about change, but not implementing change is not sustainable.

\section{Implications of Enhancing Research Evaluation Transparency}

A central tenet of our proposal is that changing how research activities are reported and evaluated in ways that explicitly recognize engagement in more robust scientific practices should increase the likelihood that researchers engage in said practices. The research philosophy statement and annotated $\mathrm{CV}$ provide a critical source of information for succinctly communicating how and why a scientist conducts his or her research in a particular manner. Incorporating these behaviors as criteria into a collectively developed evaluation system within an academic department conveys both a commitment to and formal structure for rewarding those who choose to engage in more robust scientific activities. It should be noted that these interconnected reporting and evaluation mechanisms neither result in a top-down mandate to participate in robust science nor ensure that researchers will be unable to find new or different ways to "game the system" if implemented in the manner we have recommended. However, we do believe that our proposal will make efforts to game the system less instrumental to the goals that our field see as desirable. In the end, we believe that draconian measures such as mandating open science practices are both intractable and untenable solutions for improving the quality of psychological science. Our vision has instead been to consider how we might "level the playing field" such that efforts to engage in robust science are better captured and rewarded as valuable contributions by researchers, colleagues, and the institutions/disciplines in which research occurs. We further believe that merely asking faculty to report on activities that relate to robust science can serve as a powerful nudge (Thaler \& Sunstein, 2008) for faculty to engage in those activities. In this sense, encouraging scientists to more precisely communicate how and why their research activities are conducted and encouraging departments and disciplines to transparently communicate what activities are valued has the potential to change institutionalized norms concerning participation in robust science in a more bottom-up and emergent fashion.

An important implication of our envisioned system-and one reason we believe it has the potential to be successful-is that it allows individual researchers and departments to more easily and transparently assess person-organization fit and use that information to inform critical decisions and/or functions. Person-organization fit can generally be defined as "the compatibility between people and organizations that occurs when:

(a) at least one entity provides what the other needs,

(b) they share similar fundamental characteristics, or (c) both" (Kristof, 1996, p. 4). ${ }^{6}$

\footnotetext{
${ }^{6}$ The meaning conveyed by part (a) of this definition is sometimes used as the definition of person-job fit to more specifically refer to the match between an individual's abilities and
} 
Person-organization fit features prominently in many theories of organizational culture, climate, and change, and has been shown to correlate with a variety of desirable outcomes (e.g., job performance, satisfaction, commitment, turnover, etc.; Kristof-Brown, Zimmerman, \& Johnson, 2005). Person-organization fit also plays a central role in one of the most popular and commonly studied frameworks for understanding organizational culture and its emergence over time - the attraction-selection-attrition framework (Schneider, 1987; Schneider, Goldstein, \& Smith, 1995). The attraction-selection-attrition framework holds that people are attracted to organizations with congruent goals, values and/or desired resources; organizations select individuals for similar reasons; and individuals voluntarily (or involuntarily) leave organizations if they perceive a lack of fit or a better fit can be found elsewhere. The attraction-selection-attrition framework further highlights the significance of socialization and feedback processes that enable individuals and organizations to express values, goals, and expectations in this process. The information gleaned from such interactions helps both individuals and organizations identify whether or how to adapt to incongruences (top-down change) or establish alternative criteria/agreements (bottom-up change) in an effort to maintain person-organization fit.

We believe the research reporting and evaluation system we have proposed enables a more accurate and transparent assessment of the fit between researcher and institution that is likely to promote (but not mandate) participation in robust science through a similar attraction-selection-attrition process. For example,

preferences/needs and the demands required of and rewards provided by one's primary job tasks. For purposes of the present discussion, it is not necessary to distinguish between these different conceptualizations and simply note that we are referring to the overall compatibility between an individual and his or her place of work. appointment and hiring decisions could be made more effectively and efficiently if departments communicate their values and expectations concerning the robust research behavior (e.g., in the form of a Multi-Attribute Utility analysis) they are looking for in potential applicants and if applicants provide evidence of the likelihood to carry out such behaviors (e.g., via an annotated $\mathrm{CV}$ and research philosophy). Performance evaluation and feedback, such as they are in academia, could also benefit from a more transparent reporting and evaluation mechanism. For example, in pre-tenure reviews, a junior faculty member and his or her more senior advisors can evaluate the extent to which the research being carried out by the junior member reflects how members of a department define robust science. To the extent a department decides to value particular robust scientific practices (e.g., collecting larger samples, openly sharing data, etc.), concrete recommendations can be provided that are presumably "safer" for the junior colleague given its implied support by the remainder of the faculty. The annotated CV, research philosophy, and Multi-Attribute Utility evaluation system would also provide the opportunity for faculty members to identify the extent to which their own research behaviors and values are compatible with those expressed by their colleagues, which could carry implications for decisions related to retention for an individual faculty member and the department.

In addition to the effects of a more transparent research reporting and evaluation system on key department functions, there is also the potential for changes to a departmental culture's concerning robust science practices. For example, asking researchers to include metrics regarding sample sizes, data sharing/transparency, sensitivity analyses, or replication efforts (among others) on an annotated $\mathrm{CV}$ and incorporating such items as part of one's evaluative/Multi-Attribute Utility system should help promote a broader definition of reproducibility. Though it is commonly assumed that direct replication is the gold standard for ensuring accurate scientific inferences, there are numerous ways in which a more robust and 
reproducible science can be promoted (e.g., conducting more highly powered studies, testing model assumptions, sharing data, etc.; Goodman et al., 2016 ). Any steps taken to continue moving this needle in a positive direction will contribute to the confidence in and rigor of individual studies, and in psychological science more generally. The proposed changes to a department's research reporting and evaluation procedures may also make it more likely for faculty to transmit norms regarding robust science to undergraduates, graduate students, and postdocs who both assist with and carry out their own research. Moreover, we believe that faculty who read other people's philosophies and annotated CVs will be inspired to change their own approach to research. The majority of knowledge regarding the research enterprise and how to conduct scientific research in academia is still transmitted idiosyncratically and informally through interaction with peers as well as direct advising and role modeling from faculty researchers to their junior collaborators (National Academies of Sciences, Engineering, \& Medicine, 2017). Enacting systems and shared norms that robust scientific practices are valued in one's research thus communicates to both current and future generations of psychological scientists that such practices are important elements of a scientists' tasks and responsibilities.

There are, of course, potential costs to adopting our proposal. One potential cost is added time and effort in evaluating dossiers that include more information than has typically been captured on the traditional $\mathrm{CV}$. Most faculty are familiar with the traditional citation format and therefore may feel more comfortable making evaluations based on publication counts, perceived journal reputation, citation counts, and the like. It is true that providing more information may make evaluations more cumbersome, however we believe the added time and effort is a worthy cost if it leads to a more transparent evaluation process that explicitly recognizes and incentivizes robust scientific practices. Further, we suspect that pairing the annotated $\mathrm{CV}$ and research philosophy with a Multi-Attribute Utility analysis will ultimately streamline and help to standardize the evaluation process.

A second potential concern is whether the proposed changes might negatively affect one's career trajectory or mobility. For instance, conducting research that emphasizes reproducibility in a department that eschews it (or vice versa) could restrict upward mobility. Alternatively, one may develop a research philosophy and agenda to fit one university, but find that it does not fit well with other desirable institutions. While these scenarios are certainly possible, we do not believe these concerns are new; further, we would argue that the research philosophy provides a platform for one to not just articulate his or her current philosophy, but also how it has changed or will change. The value of a research philosophy is not merely as a statement of one's core values, but rather that it can serve as a bridge between the institutional values and one's actual approach to research (as documented on the annotated $\mathrm{CV}$ ).

A third potential concern is how the added information will be received and reviewed at higher-levels of administration. While we recognize that there is some uncertainty with how the annotated $\mathrm{CV}$ and research philosophy will be viewed by college and campus administrators and promotion and tenure committees, we do not believe that this uncertainty is insurmountable. Research institutions, administrators, and faculty all have a vested interest in promoting reproducible science. Based on our own experience at the University of Maryland, we suspect that administrative officials will be surprisingly receptive to these proposed changes or variants thereof. Indeed, part of exacting bottom up change in how faculty are reviewed requires that faculty push for change.

\section{DISCUSSION}

In a recent paper in Nature, Jeffrey Flier (2017) argued that reproducibility and robustness are under-appreciated in decisions regarding appointment, tenure, and promotion of faculty. Flier (2017) also suggested that greater emphasis be placed on 'critical self-reflection', an increase in 
institutional incentives to increase transparency and data sharing, and better metrics for evaluating research publications. These recommendations have been echoed in various reports. The National Academy of Science Engineering and Medicine (2018) recommended that research institutions explicitly recognize and reward open-science and reproducibility efforts and place more value on research integrity when evaluating faculty for tenure and promotion ; likewise, the National Association of Scholars argued that "Scientists must reform the professional incentives that reward inadequate research and punish the unglamorous but essential work of checking research that has already been done. Researchers should perform more replication studies and accord greater esteem to research that produces negative results. Professional organizations, journals, and university tenure and promotion committees must all commit themselves to support these changes" (pg. 47, Randall \& Welser, 2018). Our proposed reforms are consistent with these suggestions while directly connecting back to the the Transparency and Openness Promotion journal publication standards promoted by Nosek et al. (2015). There is no one-size-fits all approach to science, and instituting change is not as easy as setting institutional mandates and expecting faculty to fall in line. We advocate for an approach that asks institutions to set broad guidelines, but allow individual faculty the flexibility to set an agenda that explicates their own values and provide a mechanism for transparently reporting and evaluating the details of specific pieces of scholarship.

Though the research philosophy and annotated $\mathrm{CV}$ are documents created by individual faculty, both documents should be informed by institutional values. Indeed, we suggest that departments develop reporting dimensions included in the article details section of the annotated $\mathrm{CV}$ that have a clear connection to criteria that can be useful for evaluating tenure and promotion (see Box 3 for some suggestions). This is not to say that each dimension will relate directly to some institutional criterion, but rather that the collection of criteria will permit a more holistic evaluation of individual pieces of scholarship. The holistic evaluation should, in principle, enable one to better judge the contribution of individual publications and nudge evaluators away from relying too heavily on number of publications, impact factors, or journal reputations, and instead rely more on factors like effort, workload, rigor, reproducibility, and scope of scientific contribution (cf. Hicks et al., 2015). Departments may still desire to use number and impact factors as part of the evaluation process; however, we believe that these dimensions are best viewed alongside information about how researchers conduct their scientific efforts. Because there are many ways in which good science can be conducted, having a more complete and balanced way of describing and evaluating one's research activities should incentivize good scientific behavior. 


\section{Box 3. Recommendations for creating a research philosophy \& annotated CV}

Research Philosophy: What should your research philosophy look like? We suggest a relatively short statement that:

(1) Clearly articulates a set of core values. These may or may not include your position along the dimensions described above (e.g., Box 1), but definitely should indicate the aspects of your own approach to research that you consider most important. Your philosophy need not address every nuance: Focus on those elements that form your core values.

(2) Clearly articulates the focus of inquiry and scope of your research program. This is perhaps the most similar to content in the more typical "research statement", but certainly the topics and focus of one's research is an important part of the research philosophy. Detailed descriptions of the program of research and future directions are important, but perhaps best saved for the broader statement of research.

(3) Includes concrete examples. A research philosophy is less valuable if it simply reiterates a set of 'buzzwords', instead it should include specific illustrations of your research philosophy and priorities. Details and examples can help, and note that these need not be examples from your own research. For example, if you value open science practices but have not yet made the transition yourself, you can still articulate this as one of your core values and point to specific examples of your 'aspirational' practices (e.g., McKiernan, 2017).

(4) Reflects how one's work strives to achieve institutional or departmental values.

(5) Links to the dimensions reflected on the annotated CV.

Annotated $\boldsymbol{C} \boldsymbol{V}$ : We suggest that faculty within departments work collaboratively to develop a format for an annotated CV that:

(1) Includes reporting criteria that are informed by your institution's values and disciplinary norms.

(2) Includes elements that are easy to report, as objective as possible, and verifiable.

(3) Provides an opportunity for faculty to contextualize (i.e., increase transparency, provide information for evaluators) their research contributions. This is important in part because research in the psychological and brain sciences is incredibly diverse in terms of content, norms for sample sizes, applicability of research to applied problems, theoretical orientation, methodology, and even where the lead author is placed in the authorship list. This diversity makes it difficult for non-experts in a field to interpret the scope of individual pieces of scholarship. 
The replication crisis within psychology and other fields has brought to light the need to both incentivize and encourage the conduct of reproducible research. We argue that the creation of research philosophy statements and the use of annotated CVs can play an important role in nudging scientists toward better research practices both by encouraging scientists to articulate their scientific values more fully and by allowing transparent reporting of their efforts. While many scientists support the open-science movement, we have seen relatively little discussion regarding how to document and incentivize the efforts of scientists who choose to use open-science practices. Likewise, while we suspect that many scientists value the concept of replication, replication efforts are often either not published or documented on the $\mathrm{CV}$. Encouraging scientists to report reproducibility efforts within the annotated $\mathrm{CV}$ can both (a) incentivize researchers to do more to ensure that their work is reproducible, even when these efforts are not ultimately included within publications, and (b) enable colleagues to better evaluate the scope of one's research and the degree to which his or her work adheres to departmental, university, or disciplinary standards.

\section{Summary}

Conducting reproducible science is more than following along with a set of definitions. It requires concrete actions that involve setting standards for statistical power for one's own research, disclosing which analyses were planned a priori and which were decided after the data were observed, ensuring that statistical analyses are appropriate and justified, and permitting others to verify one's work. Part of incentivizing these actions involves the promise that the efforts can be documented and that they will be recognized as part of one's scholarly activity in high-stakes decisions such as tenure and promotion and merit-pay increases. The research philosophy and annotated $\mathrm{CV}$ permit one to document these efforts transparently.
We believe that the research philosophy and annotated $\mathrm{CV}$ will be useful to both individual faculty and departments. For individual faculty members, the research philosophy allows one to set priorities and evaluate progress. It enables self-reflection and permits one to make his or her own priorities and goals public. For example, job applicants can be evaluated both by what they have accomplished and by examining how their values fit with institutional or departmental values. If your department values basic science more than applied science (or vice versa), then it may be useful to know how individual job candidates prioritize their work along this dimension. If your department explicitly seeks to promote open science, then hiring people who espouse this view may be a priority. The annotated $\mathrm{CV}$ also can be useful for departments in evaluating faculty workload, making decisions about merit pay, as well as pre-tenure reviews.

Our proposal can be summed up in one sentence: Give faculty a voice in deciding how their work should be evaluated and allow them to report details of their scholarship relevant to their evaluation. We suspect that a great majority of scientists would, if given a choice, prefer a system that allows them to focus more on ensuring that their work is reproducible over one in which the standard for tenure are based on an ever increasing threshold for quantity. Departments will still need to have a say in the values they espouse, but giving faculty a voice and allowing them to focus on some but not all dimensions will, in our opinion, go a long way toward changing a culture from one that relies on the quantity as an index of productivity to one that places more emphasis on quality. 


\section{References}

Agnoli, F., Wicherts, J. M., Veldkamp, C. L., Albiero, P., \& Cubelli, R. (2017). Questionable research practices among italian research psychologists. PloS One, 12(3), e0172792.

Aguinis, H. (2013). Performance management (3rd ed.). Upper Saddle River, NJ: Prentice Hall/Pearson Education.

Aguinis, H., Joo, H., \& Gottfredson, R.K. (2011). Why we hate performance management--and why we should love it. Business Horizons, 54, 503-507.

Arruda, J.R.F., Champieux, R., Cook, C., Davis, M.E.K., Gedye, R., Goodman, L., Jacobs, N., Ross, D., \& Taylor, S. (2016). The journal impact factor and its discontents: steps toward responsible metrics and better research assessments. Open Scholarship Initiative Proceedings, $\quad 1, \quad 1-8 . \quad$ DOI: http://dx.doi.org/10.13021/G88304

Ball, P. (2011). Are scientific reputations boosted artificially? Nature, Published online May 6, 2011. doi:10.1038/news.2011.270

Beck, J. W., Beatty, A. S., \& Sackett, P. R. (2014). On the distribution of job performance: The role of measurement characteristics in observed departures from normality. Personnel Psychology, 67, 531-566.

Cameron, K.S., \& Quinn, R.E. (2011). Diagnosing and changing organizational culture: Based on the Competing Values Framework (3rd ed.). San Francisco, CA: Jossey-Bass.

Campbell, J. P. (1990). Modeling the performance prediction problem in industrial and organizational psychology. In M. Dunnette \& L. M. Hough (Eds.), Handbook of industrial and organizational psychology (Vol. 1, pp. 687-731). Palo Alto, CA: Consulting Psychologists Press.

Caon, M. (2017). Gaming the impact factor. Where who cites what, whom, and when. Australasian Physical \& Engineering Sciences in Medicine,
40, $273 \quad-276$.

https://doi.org/10.1007/s13246-017-0547-1

Chakravartty, P., Kuo, R., Grubbs, V., McIlwain, C. (2018). \#CommunicationSoWhite, Journal of Communication, $\quad 68, \quad 254-266$, https://doi.org/10.1093/joc/jqy003

Den Hartog, D.N., Boselie, P., \& Paauwe, J. (2004). Performance management: A model and research agenda. Applied Psychology: An International Review, 53, 556-569.

Ebersole, C. R., Axt, J. R., \& Nosek, B.A. (2016). Scientists' reputations are based on getting it right, not being right. PLoS Biology 14(5), e1002460.

https://doi.org/10.1371/journal.pbio.1002460

Edwards, M.A. \& Roy, S. (2017). Academic research in the $21^{\text {st }}$ century: Maintaining scientific integrity in a climate of perverse incentives and hypercompetition. Environmental Engineering Science, 34, 51-61.

Flier, J. (2017). Faculty promotion must assess reproducibility. Nature News, 549(7671), 133.

Foster-Fishman, P.G., Nowell, B., \& Yang, H. (2007). Putting the system back into systems change: A framework for understanding and changing organizational and community systems. American Journal of Community Psychology, 39, 197-215.

Fowler, J. H., \& Aksnes, D. W. (2007). Does self-citation pay? Scientometrics, 72 (3), 427-437 DOI: $10.1007 / \mathrm{s} 11192-007-1777-2$

Grand, J.A., Rogelberg, S.G., Allen, T.D., Landis, R.S., Reynolds, D., Scott, J.C., Tonidandel, S., \& Truxillo, D.M. (2018). A systems-based approach to fostering robust science in Industrial-Organizational psychology. Industrial and Organizational Psychology: Perspectives on Science and Practice, 11, 4-42.

Goodman, S. N., Fanelli, D., \& Ioannidis, J. P. A. (2016). What does research reproducibility mean? Science Translational Medicine, 8, 341ps12. DOI: 10.1126/scitranslmed.aaf5027

Hicks, D., Wouters, P., Waltman, L., de Rijcke, S., \& Rafols, I. (2015). The Lieden Manifesto for research metrics. Nature, 520, 429 - 431. 
Higginson A.D. \& Munafò, M.R. (2016) Current Incentives for Scientists Lead to Underpowered Studies with Erroneous Conclusions. PLOS Biology 14(11), e2000995.https://doi.org/10.1371/journal.pbio.2 $\underline{000995}$

John, L. K., Loewenstein, G., \& Prelec, D. (2012). Measuring the prevalence of questionable research practices with incentives for truth telling. Psychological Science, 23(5), 524-532.

Kerr, S. (1975). On the folly of rewarding A, while hoping for B. Academy of Management Journal, 18, 769-783.

Kidwell, M. C., Lazarevic, L. B., Baranski, E., Hardwicke, T. E., Piechowski, S., Falkenberg, L-S., Kennett, C., Slowik, A., Sonnleitner, C., Hess-Holden, C., Errington, T. M., Fiedler, S., \& Nosek, B. A. (2016). Badges to acknowledge open practices: A simple, low-cost, effective method for increasing transparency. PLOS Biology, 14, e1002456. Doi: 10.1371/journal.pbio.1002456

Knobloch-Westerwick, S., Glynn, J.C., \& Huge, M. (2013). The Matilda effect in science communication: An experiment on gender bias in publication quality perceptions and collaboration interests. Science Communication, $35,603-625$.

Kristof, A. L. (1996). Person-organization fit: An integrative review of its conceptualizations, measurement, and implications. Personnel Psychology, 49, 1-49.

Kristoff-Brown, A.L., Zimmerman, R.D., \& Johnson, E.C. (2005). Consequences of individuals' fit at work: A meta-analysis of person-job, person-organization, person-group, and person-supervisor fit. Personnel Psychology, 58, 281-342.

Lewin, K. (1947). Frontiers in group dynamics: Concept, method and reality in social science; social equilibria and social change. Human relations, 1, 5-41.

Maliniak, D., Powers, R., \& Walter, B.F. (2013). The gender citation gap in international relations. International Organization, 67, 889-922.

McKiernan, E. C. (2017). Imagining the "open" university: Sharing scholarship to improve research and education. PLOS Biology, 15(10), e1002614.

https://doi.org/10.1371/journal.pbio.1002614

Randall, D. \& Welser, C. (2018). The Irreproducibility Crisis of Modern Science: Causes, Consequences, and a Road to Reform. National Association of Scholars.

National Academies of Sciences, Engineering, \& Medicine. (2017). Fostering Integrity in Research. Washington, DC: The National Academies Press.

National Academies of Sciences, Engineering, \& Medicine. (2018). Open Science By Design: Realizing a Vision for 21st Century Research. Washington, DC: The National Academies Press. [uncorrected proof cited]

Nosek, B. A., Spies, J. R., \& Motyl, M. (2012). Scientific utopia: II. Restructuring incentives and practices to promote truth over publishability. Perspectives on Psychological Science, 7(6), 615-631.

Nosek, B. A., Alter, G., Banks, G. C., Borsboom, D., Bowman, S. D., Breckler, S. J., Buck, S., Chambers, C. D., Chin, G., Christensen, G., Contestabile, M., Dafoe, A., Eich, E., Freese, J., Glennerster, R., Goroff, D., Green, D. P., Hesse, B., Humphreys, M., Ishiyama, J., Karlan, D., Kraut, A., Lupia, A., Mabry, P., Madon, T. A., Malhotra, N., Mayo-Wilson, E., McNutt, M., Miguel, E., Levy Paluck, E., Simonsohn, U., Soderberg, C., Spellman, B. A., Turitto, J., VandenBos, G., Vazire, S., Wagenmakers, E. J., Wilson, R., \& Yarkoni, T. (2015). Promoting an open research culture. Science, 348, 1422-1425. Doi: 10.1126/science.aab2374

Smaldino, P.E., \& McElreath, R. (2016). The natural selection of bad science. Royal Society Open Science, 3, 160384. http://dx.doi.org/10.1098/rsos.160384

Schneider, B. (1987). The people make the place. Personnel Psychology, 40, 437-453. 
Schneider, B., Goldstein, H.W., \& Smith, D.B. (1995). The ASA framework: An update. Personnel Psychology, 48, 747-773.

Schwarz, G.J. \& Kennicutt, R.C. Jr. (2004). Demographic and Citation Trends in Astrophysical Journal papers and Preprints https://arxiv.org/abs/astro-ph/0411275v1

Simon, H.A. (1956). Rational choice and the structure of the environment. Psychological Review, 63, 129-138.

Thaler, R. H. \& Sunstein, C. R. (2008). Nudge: Improving Decisions about Health, Wealth, and Happiness. New Haven: Yale University Press.

Yu, E. C., Sprenger, A. M., Thomas, R. P., \& Dougherty, M. R. (2014). When heuristics and science collide. Psychonomic Bulletin and
Review, $\quad 21, \quad 268-82 \quad$ DOI: 10.3758/s13423-013-0495-z

Verma, I. M. (2015). Impact, not impact factor. Proceedings of the National Academy of Sciences, 112(26), 7875-7876.

Viswesvaran, C., \& Ones, D. S. (2000). Perspectives on models of job performance. International Journal of Selection and Assessment, 8, 216-226.

Weick, K.E. (1976). Educational systems as loosely coupled systems. Administrative Science Quarterly, 21, 1-19.

Weick, K.E. (1995). Sensemaking in organizations (Vol. 3). Thousand Oaks,CA: Sage. 